\section{Estimación del presupuesto de estructura y planta propulsora de un buque tipo Suezmax}

\author{
Carlos Mascaraque-Ramírez ${ }_{a, b}{ }^{1 \oplus}$, Cristian \\ Alcántara-Reinaldos ${ }^{10}$, Pedro Gil-Padilla1 ${ }^{10}$, \\ Elisa Uriarte-Fernández ${ }^{10}$, Lorena Para \\ González2 \\ 1 Universidad Politécnica de Cartagena \\ ${ }^{2}$ Centro Universitario de la Defensa en la Academia General del Aire \\ ${ }^{1}$ carlos.mascaraque@upct.es; ${ }^{1}$ cristian_181092@hotmail.com; \\ 1pedrogilpadilla@gmail.com; ${ }^{1}$ elisauriartef@gmail.com; \\ 2lorena.para@cud.upct.es \\ DOI https://doi.org/10.33412/pri.v9.1.2053
}

\section{(잉(-)}

Resumen: En los proyectos de nueva construcción de buques de grandes dimensiones, como es el caso de los petroleros tipo Suezmax, es de gran importancia realizar un presupuesto en fases muy tempranas y con poca información disponible. En este aspecto, la presente investigación estudia un posible método de cálculo para los dos conceptos más significativos en el presupuesto de un buque, que son la estructura y la planta propulsora, clasificadas como grupos, 100 y 200 según los manuales técnicos empleados por la US Navy. La formulación y consideraciones necesarias serán expuestas, los resultados obtenidos muestran la relevancia de estos conceptos frente al presupuesto total y por lo tanto, la necesidad de definir un método lo más exacto posible en las etapas tempranas del proyecto.

Palabras clave: construcción naval, Suezmax, presupuesto.

Title: Budget estimation for the structure and propulsion plant of a Suezmax ship.

\begin{abstract}
In the case of new projects for the construction of large vessels, such as Suezmax tankers, it is of great importance to make a budget in very early stages and with little information available. In this regard, the present research studies a possible method of calculation for the two most significant concepts in a ship's budget, namely structure and propulsion plant, classified as groups 100 and 200 according to the technical manuals used by the US Navy. The necessary formulation and considerations will be presented, and the results obtained, in the case of a study of a Suezmax vessel, will be presented in order to validate the processing used.
\end{abstract}

Key words: shipbuilding, Suezmax, budget.
Tipo de artículo: original

Fecha de recepción: 10 de marzo de 2018

Fecha de aceptación: 17 de octubre de 2018

\section{Introducción}

La estimación presupuestaria de los grandes buques navales en las etapas iniciales de sus proyectos, se caracteriza por ser una labor compleja y con un gran impacto sobre el correcto desarrollo del mismo. Un erróneo cálculo inicial de presupuesto puede conllevar la pérdida de contratos, debido a la falta de competitividad, es decir a los elevados precios frente a la competencia, o por el contrario comprometerse con el cliente a unos costes demasiado bajos, y por lo tanto, sean imposibles de cumplir, lo que tendría como resultado unas pérdidas inasumibles para la empresa.

Diversos autores han desarrollado formulaciones aplicables a diferentes buques mercantes para la estimación de su presupuesto [1], centrándose en las dimensiones principales del buque $y$ formulando unas ecuaciones que permiten estimar los grandes grupos de coste que conforman el proyecto. También se ha investigado en el cálculo de las horas necesarias para la ejecución de los trabajos, tanto en los talleres, como en los diques $[2,3]$.

Si bien otro aspecto primordial para comenzar el proceso de cálculo del presupuesto de un proyecto de la envergadura de un buque, es la división de este en diferentes conceptos. Por esto, las publicaciones más relevantes sobre gestión de proyecto $[4$, 5] proponen una distribución estándar para todo proyecto. En el campo naval, la US Navy [6] ha publicado un manual en el que especifica los grupos de coste más idóneos para los grandes buques de guerra, pudiendo aplicarse a los buques mercantes 0 de recreo.

El presente artículo de investigación se centra en el cálculo de dos conceptos presupuestarios, que son las partidas definidas por la US Navy [6], 100 "Estructura del casco" y 200 "Planta propulsora", la elección de estas dos partidas se debe a que usualmente son las que tienen mayor impacto sobre el presupuesto total del buque, llegando hasta un $75 \%$ del presupuesto final de materiales y equipos del buque, sin ser considerada la mano de obra.

\section{Metodología y caso de estudio}

Este artículo se centra en el estudio de embarcaciones del tipo Suezmax. Los petroleros de la clase Suezmax tienen como propósito el transporte de crudo y derivados del mismo, carga que se caracteriza por su alta densidad aparente 0 bajo coeficiente de estiba. El término Suezmax se utiliza para denominar a los petroleros cuyas dimensiones máximas son las que el canal de Suez permite, donde la autoridad del canal limita las dimensiones de los buques a: $20,1 \mathrm{~m}$ de calado con mangas inferiores a 50 metros y 12,2 metros de calado para mangas comprendidas entre los 50 y los 77,5 metros. 
El caso concreto de estudio, es una embarcación con las siguientes características:

- Eslora máxima: 264,6 m

- Manga máxima: $49 \mathrm{~m}$

- Calado de trazado: $15 \mathrm{~m}$

- Puntal a la cubierta principal: $24,5 \mathrm{~m}$

- Coeficiente de bloque: 0,858

- Desplazamiento: 174.349 Ton.

Una vez conocido el buque del que se va a calcular el presupuesto, se procede a analizar por separado el grupo 100 "Estructura del casco" y el grupo 200 "Planta propulsora".

\subsection{Grupo 100: estructura del casco}

Este grupo engloba todo lo referente a la estructura del buque, es decir, casco, refuerzos y elementos auxiliares asociados a la misma. Se ha dividido en diferentes conceptos de acuerdo con lo establecido en los manuales de US Navy [6]. Aunque estos manuales sean de aplicación para buques militares, investigaciones recientes han verificado su utilización para embarcaciones mercantes [7], como es el caso de estudio planteado en este trabajo.

El grupo 100 "Estructura del casco" se separa en los siguientes subconceptos:

- Acero laminado.

- Piezas fundidas y forjadas.

- Timones y accesorios.

- Materiales auxiliares de construcción del casco.

- Preparación de superficies.

- Coste de pintado del buque.

- Coste de pinturas de tuberías.

- Galvanizado y cementado.

- Protección catódica.

Las fórmulas utilizadas para el cálculo de las diferentes partidas se muestran a continuación, donde se definirá posteriormente cada uno de los valores necesarios y se introducirá una aproximación para su cálculo. Estas fórmulas han sido desarrolladas a partir de estudios previos e investigaciones de diversos autores, adaptándolas a los valores actuales de referencia de los buques modernos $[2,8]$.

Acero laminado:

$$
C_{A L}=c c s \cdot c a s \cdot p s \cdot W S T
$$

Timones y accesorios:

$$
C_{\text {TIM }}=2 \cdot 40 \cdot L_{\text {timón }}^{2} \cdot H_{\text {timón }}
$$

Preparación de superficies:

$$
C_{\text {sup }}=\left(S_{O V}+S_{O M}+S_{C I}+S_{M C}\right) \cdot P_{\text {sup }}
$$

Coste de pinturas de tuberías:

$$
C_{\text {pinttub }}=0.18 \cdot(0.057 \cdot B H P+0.18 \cdot L) \cdot K
$$

Piezas fundidas y forjadas:

$$
C_{F F}=4 \cdot L \cdot H
$$

Materiales auxiliares de construcción del casco:

$$
C_{\text {Aux }}=W S T \cdot 0,1 \cdot c c s \cdot c a s \cdot p s
$$

Coste de pintado del buque:

$$
C_{\text {pint }}=P_{p S o v} \cdot S_{O V}+P_{p S o m} \cdot\left(S_{O M}+S_{C I}+S_{M C}\right)
$$

Galvanizado y cementado:

$$
C_{\text {galv } / \mathrm{cem}}=0.07 \cdot\left(C_{\text {pint }}+C_{\text {pinttub }}\right)
$$

Protección catódica:

$$
C_{\text {cad }}=1.55 \cdot S_{O V}
$$

Para determinar los anteriores conceptos, es necesario disponer de ciertos parámetros que se desconocen en una fase tan temprana del desarrollo del proyecto, por lo que se han de estimar partiendo de otros conceptos conocidos en la fase de anteproyecto.

Con esta finalidad, se desglosan los parámetros utilizados para el cálculo de las partidas citadas:

- El peso del acero (WST) del buque ha sido calculado de acuerdo con la fórmula aportada, a tal efecto, en las investigaciones de Alvariño-Castro et. al [1], y que depende de las dimensiones principales del buque.

- La eslora ( $L_{\text {timón }}$ y la altura del timón ( $\left.H_{\text {timón }}\right)$, se han estimado estableciendo una correlación entre la eslora de un buque conocido, las dimensiones requeridas del timón correspondiente al mismo, y finalmente, extrapolando el valor para la eslora de nuestro buque.

- Se toma como precio de la tonelada de acero el valor actual de mercado, que corresponde para el tercer trimestre de 2017 a $860 €$ por tonelada.

- Respecto al precio del pintado del buque, ha sido necesario calcular la superficie de obra viva, obra muerta, interior del casco, cubiertas y mamparos. Para todas estas superficies se ha partido de formas geométrica sencillas, es decir de prismas rectangulares con las dimensiones más básicas del buque, eslora, manga, calado y puntal. Sobre ellas las superficies de estas formas sencillas, se han aplicado coeficientes de corrección según las formas de la embarcación y su cuaderna maestra.

\subsection{Grupo 200: planta propulsora}

Este grupo se desglosa en las siguientes partidas presupuestarias:

- Motores propulsores.

- Línea de ejes.

- Hélices propulsoras.

- Resto de la planta propulsora. 
Las fórmulas utilizadas para el cálculo de las diferentes partidas se muestran a continuación:

Motores propulsores:

$$
C_{\text {MOT }}=\mathrm{cmd} \cdot N \cdot N_{c} \cdot 0.85 \cdot \frac{D I A^{2.2}}{R P M^{0.75}}
$$

Línea de ejes, acoplamientos:

Línea de ejes, reductores:

$$
C_{A C O P}=\operatorname{cacop} \cdot \frac{B H P}{R P M}
$$

$$
C_{R E D}=\mathrm{cred} \cdot \operatorname{Pr}^{0.5}
$$

Línea de ejes, chumaceras:

$$
C_{\text {CHUM }}=\mathrm{cchum} \cdot \mathrm{BHP}
$$

Línea de ejes, bocinas y cierres:

$$
C_{B Y C}=c b y c \cdot B H P^{0.85}
$$

Hélices propulsoras:

$$
C_{H E L}=\text { chel } \cdot B H P^{0.7}
$$

Resto de la planta propulsora:

$$
C_{p p}=W E R \cdot c c s \cdot c a s \cdot p s \cdot c p e
$$

En las partidas arriba citadas han intervenido determinadas características del buque, como en el caso del grupo de estructura del casco, ha sido necesario estimar.

- La potencia al freno se ha tomado del buque de referencia, y se ha buscado en un catálogo de motores marinos de dos tiempos, uno con una potencia inmediatamente superior. La potencia instalada se corresponde con un motor de $15480 \mathrm{~kW}$ a 90 revoluciones por minuto, del fabricante MAN B\&W [9].

- No se ha considerado el coste de reductores, debido a que la hélice va directamente acoplada al motor.

- El concepto referente al resto de la planta propulsora se ha extrapolado a partir de buques similares al buque de estudio.

\section{Resultados}

En este apartado se mostrarán los resultados de aplicar los valores de los parámetros del buque de estudio, sobre la formulación expuesta, buscando la obtención de un presupuesto optimizado y de alta fiabilidad, el cual permita al astillero mejorar su competitividad empresarial [10], así como realizar un correcto seguimiento de la ejecución de la construcción del buque por técnicas de la gestión de proyectos $[4,5]$.

Con ello se aplican las ecuaciones anteriores al buque de

\begin{tabular}{|c|c|c|}
\hline Descripción & Variable & Valor \\
\hline Acero laminado & $\mathrm{C}_{\mathrm{AL}}$ & $19.550 \mathrm{k} €$ \\
\hline Coeficiente de calidad del acero & $\operatorname{ccs}$ & 0.9 \\
\hline Coeficiente de aprovechamiento del acero & cas & 1.1 \\
\hline Precio unitario del acero & ps & $860 € /$ ton \\
\hline Peso del acero del buque & WST & 22962 ton \\
\hline Timones y accesorios & $\mathrm{C}_{\mathrm{TIM}}$ & $53 \mathrm{k} €$ \\
\hline Eslora del timón & Ltimón & $7.44 \mathrm{~m}$ \\
\hline Altura del timón & $\mathrm{H}_{\text {timón }}$ & $12.01 \mathrm{~m}$ \\
\hline Preparación de superficies & $\mathrm{C}_{\text {sup }}$ & $730 \mathrm{k} €$ \\
\hline Precio por metro cuadrado & $P_{\text {sup }}$ & $12 € / \mathrm{m}^{2}$ \\
\hline Superficie obra viva & Sov & $18911 \mathrm{~m}^{2}$ \\
\hline Superficie obra muerta & Som & $6947 \mathrm{~m}^{2}$ \\
\hline Interior del casco & $\mathrm{S}_{\mathrm{Cl}}$ & $17623 \mathrm{~m}^{2}$ \\
\hline Mamparo y cubiertas & $\mathrm{S}_{\mathrm{MC}}$ & $17375 \mathrm{~m}^{2}$ \\
\hline Coste Pintura de tuberías & $\mathrm{C}_{\text {pinttub }}$ & $1 \mathrm{k} €$ \\
\hline Potencia total instalada & BHP & $20403 \mathrm{HP}$ \\
\hline Eslora & L & $264.6 \mathrm{~m}$ \\
\hline Coeficiente según pintura & K & 4.8 \\
\hline Piezas fundidas y forjadas & $\mathrm{C}_{\mathrm{FF}}$ & $26 k €$ \\
\hline Eslora & L & $264.6 \mathrm{~m}$ \\
\hline Puntal hasta la cubierta principal & $\mathrm{H}$ & $24.5 \mathrm{~m}$ \\
\hline $\begin{array}{l}\text { Materiales auxiliares de construcción del } \\
\text { casco }\end{array}$ & $\mathrm{C}_{\mathrm{AUX}}$ & $987 \mathrm{k} €$ \\
\hline Precio por tonelada de acero & ps & $860 € /$ ton \\
\hline Coeficiente de calidad del acero & $\operatorname{ccs}$ & 0.9 \\
\hline Coeficiente de aprovechamiento del acero & cas & 1.1 \\
\hline Toneladas de acero & WST & 22962 ton \\
\hline Coste pintado del buque & $\mathrm{C}_{\text {pint }}$ & $273 \mathrm{k} €$ \\
\hline Precio pintura obra viva & $\mathrm{P}_{\mathrm{pSov}}$ & $10 € / \mathrm{m}^{2}$ \\
\hline Superficie obra viva & Sov & $18911 \mathrm{~m}^{2}$ \\
\hline Precio pintura obra muerta & $P_{\mathrm{psom}}$ & $2 € / \mathrm{m}^{2}$ \\
\hline Superficie obra muerta & Som & $6947 \mathrm{~m}^{2}$ \\
\hline Interior del casco & $\mathrm{S}_{\mathrm{Cl}}$ & $17623 \mathrm{~m}^{2}$ \\
\hline Mamparo y cubiertas & $\mathrm{S}_{\mathrm{MC}}$ & $17375 \mathrm{~m}^{2}$ \\
\hline Galvanizado y cementado & $\mathrm{C}_{\text {galv/cem }}$ & $19 k €$ \\
\hline Coste pintado del buque & $C_{\text {pint }}$ & $273 \mathrm{k} €$ \\
\hline Coste Pintura de tuberías & $\mathrm{C}_{\text {pinttub }}$ & $1 \mathrm{k} €$ \\
\hline Protección catódica & $\mathrm{C}_{\mathrm{cad}}$ & $29 k €$ \\
\hline Superficie obra viva & Sov & $18911 \mathrm{~m}^{2}$ \\
\hline
\end{tabular}
estudio, obteniendo así todos los resultados que quedan recogidos y desglosados sobre la tabla 1, donde aparecen los valores de presupuesto, su definición y el valor de las variables que conforman, para cada caso, la formulación expuesta:
Tabla 1. Desglose de resultados concepto presupuestario: 100 "Estructura del casco"

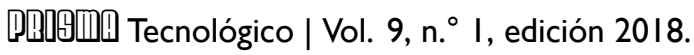


Esta partida presupuestaria asciende a un total de $21.668 \mathrm{k} €$.

En lo referente al grupo 200 "Planta propulsora", se muestran los resultados en la tabla 2.

Tabla 2. Desglose de resultados concepto presupuestario: 200 "Planta propulsora"

\begin{tabular}{|c|c|c|}
\hline Descripción & Variable & Valor \\
\hline Motores propulsores & $\mathrm{C}_{\text {MOT }}$ & $6746 \mathrm{k} €$ \\
\hline Coeficiente coste motores diésel & cmd & 20 \\
\hline Número de motores & $\mathrm{N}$ & 1 \\
\hline Número de cilindros & Nc & 6 \\
\hline Diámetro del cilindro & DIA & $720 \mathrm{~mm}$ \\
\hline Revoluciones máximas & RPM & 90 rpm \\
\hline Línea de ejes: acoplamientos & $\mathrm{C}_{A C O P}$ & $680 \mathrm{k} €$ \\
\hline Coeficiente coste acoplamientos & cacop & 3000 \\
\hline Potencia instalada & BHP & 20403 HP \\
\hline Revoluciones máximas & RPM & $90 \mathrm{rpm}$ \\
\hline Línea de ejes: reductores & $C_{\text {RED }}$ & $0 \mathrm{k} €$ \\
\hline Coeficiente coste reductores & cred & 50 \\
\hline Peso de los reductores & $\operatorname{Pr}$ & $0 \mathrm{Kg}$ \\
\hline Línea de ejes: chumaceras & $\mathrm{C}_{\text {сним }}$ & $77 \mathrm{k} €$ \\
\hline Coeficiente coste chumaceras & cchum & 3.8 \\
\hline Potencia instalada & BHP & $20403 \mathrm{HP}$ \\
\hline Línea de ejes: bocinas y cierres & $\mathrm{C}_{\mathrm{BYC}}$ & $1 \mathrm{k} €$ \\
\hline Coeficiente coste bocinas y cierres & cbyc & 7.6 \\
\hline Potencia instalada & BHP & 20403 HP \\
\hline Hélices propulsoras & C HEL & $416 \mathrm{k} €$ \\
\hline Coeficiente coste hélices & chel & 400 \\
\hline Potencia instalada & BHP & 20403HP \\
\hline Resto de la planta propulsora & $\mathrm{CPP}_{\mathrm{PP}}$ & $424 \mathrm{k} €$ \\
\hline Peso equipo restante & WER & 370 ton \\
\hline Coeficiente de calidad del acero & $\operatorname{ccs}$ & 0.9 \\
\hline $\begin{array}{l}\text { Coeficiente de aprovechamiento del } \\
\text { acero }\end{array}$ & cas & 1.1 \\
\hline Precio unitario del acero & ps & 860 €/ton \\
\hline Coeficiente comparación coste del equipo & cpe & 1.35 \\
\hline
\end{tabular}

El presupuesto total correspondiente a esta partida es de $8.344 \mathrm{k} €$.
Para comprender la importancia que tienen estas dos partidas presupuestarias sobre el presupuesto total de materiales, equipos y asistencias técnicas del buque completo, se presenta el gráfico que se muestra en la figura 1, donde se aprecia que los conceptos 100 y 200 se aproximan a un valor próximo al $75 \%$ del total del presupuesto final del buque.

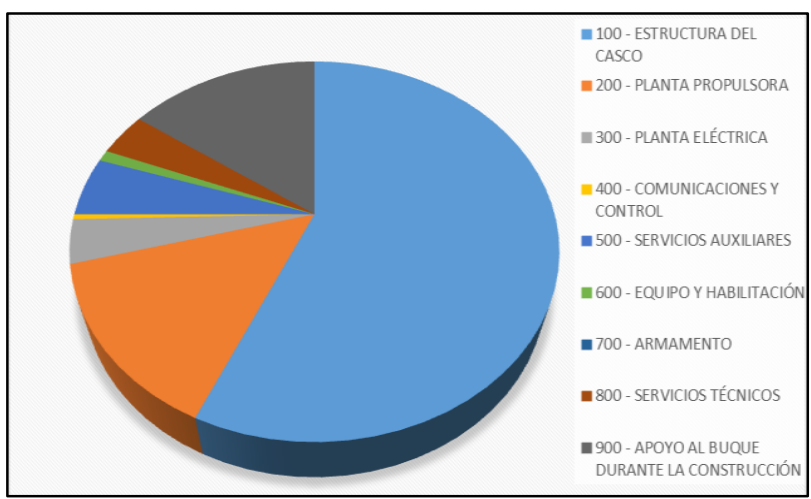

Figura 1. Descomposición de las partidas del presupuesto de un Suezmax.

En este gráfico se observa que más de un $50 \%$ del coste del buque se corresponde con el grupo 100, es por ello que cualquier mejora en el cálculo del mismo conllevará mayor precisión en el cálculo total del presupuesto de la embarcación.

Dentro del grupo 100, tal y como se observa en la figura 2, la partida correspondiente al acero laminado es la que más relevancia tiene dentro del mismo. Por lo tanto, se desprende que la correcta estimación del peso de acero del buque llevará a un presupuesto más ajustado al coste real.

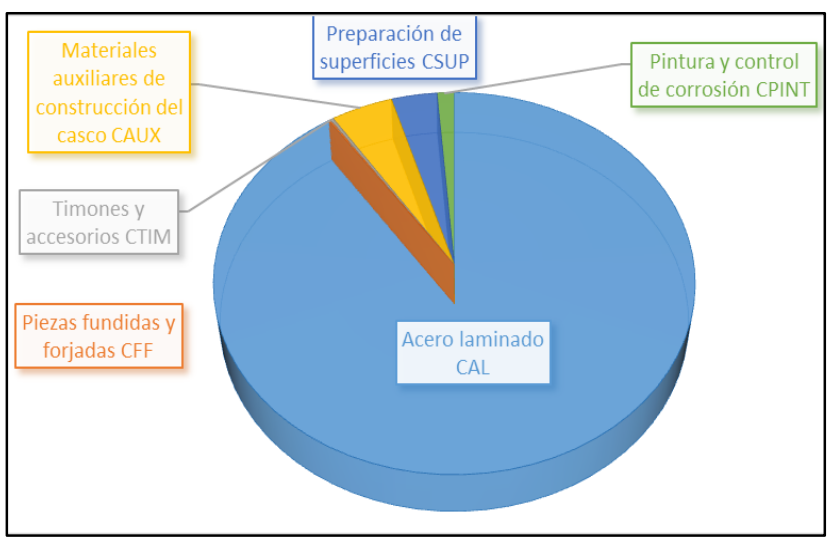

Figura 2. Descomposición de la partida 100 "Estructura del casco". 


\section{Conclusión}

Tras el estudio de los grupos 100 "Estructuras del casco" y 200" del presupuesto del buque, se pueden extraer una serie de conclusiones, siendo la más relevante, la correcta estimación de los presupuestos de un buque en las etapas más tempranas del proyecto, es crucial para la competitividad en los procesos de licitación internacional.

A pesar de la imprecisión en el cálculo del presupuesto, la bibliografía actual y la experiencia de los astilleros, indica que es mejor tener una base de referencia que no disponer de la misma. Esto permitirá a la alta dirección del proyecto, así como a los departamentos comerciales y de presupuestos, orientar la toma de decisiones relacionadas con la estimación de los distintos parámetros para el cálculo de cada partida.

Analizando el volumen que estas partidas tienen sobre el presupuesto final de las embarcaciones, se deduce que el impacto de la estimación de cálculo de estos conceptos sobre el valor final es muy elevado, resaltando la importancia de las dos partidas analizadas. Trabajando sobre estos dos conceptos, se obtendrá una alta optimización y mejora de precisión del presupuesto global final resultante.

Finalmente, se puede concluir que aparentemente resulta complicado realizar estimaciones en etapas tan tempranas de los proyectos, pero aplicando las ecuaciones presentadas en este trabajo se logra una estimación de alto valor añadido, la cual se puede aplicar para los concursos de licitación, toma de decisiones y gestión inicial de los proyectos de nuevas construcciones navales, mejorando la competitividad de la empresa en el sector naval.

\section{Referencias}

[1] USN. Naval Ships' Technical Manual. Arlington (VA): Direction of Commander, Naval Sea Systems Command, 2005.

[2] Mascaraque-Ramírez, C., L. Para-González, and D. Moreno-Sánchez "Improvement of the construction project of tuna purse seine vessels through a cost and term analysis." Ships and Offshore Structures, vol. 13, pp. 20-27, 2018.

[3] Bruce, G. and I. Garrard. The Business of Shipbuilding. New York (NY): CRC Press, 2013.

[4] Eyres, D.J. and G.J. Bruce. Ship Construction. Waltham (MA): ButterworthHeinemann, 2012

[5] Alvariño-Castro, R., J.J. Azpíroz-Azpíroz, and M. Meizoso-Fernández. El proyectobásicodelbuquemercante [The basic project of the merchant ship] Madrid, Spain: Fondo Editorial de Ingeniería Naval, 1997.

[6] B\&W, M. (2017) MAN B\&W G60ME-C9.5-TII. [Online] Available: https://marine.man.eu/applications/projectguides/2stroke/content/printed/G6 OME-C9_5.pdf. [2017 May 15]

[7] Jiang, L., E. Bastiansen, and S.P. Strandenes. "The international competitiveness of China's shipbuilding industry." Transportation Research Part E: Logistics and Transportation Review, vol. 60, pp. 39-48, 2013.

[8] PMI. A Guide To The Project Management Body Of Knowledge (PMBOK Guides). Newtown Square (PA): Project Management Institute, 2014

[9] Hermarij, J. Better Practices of Project Management based on IPMA competences. Zaltbommel, The Netherlands: Van Haren Publishing, 2013. 